\title{
No Neuroprotective Effect of Erythropoietin Under Clinical Treatment Conditions in a Rabbit Model of Escherichia coli Meningitis
}

\author{
ANNETTE SPREER, JOACHIM GERBER, MAREIKE HANSSEN, AND ROLAND NAU \\ Departments of Neurology, University Hospital, Georg-August-University of Göttingen, 37075 Göttingen, Germany
}

\begin{abstract}
Despite effective antibiotic treatment, neuronal injury is frequent among children and adults with bacterial meningitis resulting in a high rate of death and neurologic sequelae. The hematopoietic cytokine erythropoietin (EPO) provides neuroprotection in models of acute and chronic neurologic diseases. We studied whether recombinant EPO (rEPO) reduces neuronal damage in a rabbit model of Escherichia coli meningitis. Inflammation within the central nervous system (CNS) was monitored by measurement of bacterial load, pleocytosis, protein, and lactate in the cerebrospinal fluid (CSF). Neuronal damage was measured by quantification of the density of apoptotic neurons in the hippocampal dentate gyrus and the concentration of the global neuronal destruction marker neuronspecific enolase (NSE) in CSF. To increase clinical relevance, rEPO was applied as adjunctive therapy from the beginning of antibiotic therapy $12 \mathrm{~h}$ after infection. EPO treatment applied as an intravenous injection at a dose of $1000 \mathrm{IU} / \mathrm{kg}$ body weight resulted in plasma concentrations of $6993 \pm 1406 \mathrm{mIU} / \mathrm{mL}$, CSF concentrations of $1291 \pm$ $568 \mathrm{mIU} / \mathrm{mL}$, and a CSF-to-plasma ratio of $0.18 \pm 0.07$ (mean \pm SD) $6 \mathrm{~h}$ after injection. Under these treatment conditions, no antiinflammatory or neuroprotective effect of EPO was observed. (Pediatr Res 62: 680-683, 2007)
\end{abstract}

$\mathrm{I}^{\mathrm{n}}$ children and adults, bacterial meningitis continues to be associated with high rates of death and neurologic sequelae. Neuronal injury in bacterial meningitis is caused by diverse mechanisms including the local and systemic inflammatory host response to bacterial invasion and direct toxicity of bacterial compounds (1).

The hematopoietic cytokine erythropoietin (EPO) is a major component of the endogenous tissue-protective system. In the adult brain, EPO and EPO receptors are constitutively expressed only at a low level but are rapidly up-regulated as a response to metabolic stress of neurons (2) and provide neuroprotection in a multimodal manner including the Jak2 (3) and the NFkB (4) systems. Extrinsic application of human recombinant erythropoietin (rEPO) has been shown to be neuroprotective in various models of acute and chronic neurologic diseases, including ischemic stroke, chronic neuroinflammatory disorders such as experimental autoimmune encephalitis (5), and a rabbit model of subarachnoid hemorrhage (6). It acts in an antiapoptotic (3), antioxidative $(7,8)$, anti-

Received June 12, 2007; accepted July 21, 2007.

Correspondence: Roland Nau, M.D., Department of Neurology, University Hospital, Georg-August-University of Göttingen, Robert-Koch-Str. 40, 37075 Göttingen, Germany; e-mail: rnau@gwdg.de

This work was supported by the Else Kröner-Fresenius-Stiftung. inflammatory $(9,10)$, and glutamate-inhibitory (11) manner. Since neuronal injury in bacterial meningitis is mediated by inflammation, reactive oxygen species and toxicity of excitatory amino acids, these properties of rEPO appeared promising with respect to a neuroprotective effect of rEPO in the therapy of this disease. EPO is a body's own hormone and rEPO has been applied for long time in the therapy of different types of anemia. The side effects of rEPO are limited and mainly occur during long-term administration. As bacterial meningitis is an acute infection, a potentially neuroprotective treatment would be administered for a short period and should be well tolerated. Therefore, EPO appeared to be an ideal candidate for adjunctive treatment in bacterial meningitis.

The earliest possible starting point for adjunctive therapy in the treatment of patients suffering from bacterial meningitis is the time of admission to a medical center. A multitude of experimental studies have been published demonstrating a neuroprotective effect of the investigated pharmaceutical compounds in animal models, when applied before the impairing event (e.g. ischemic stroke) yet failing to be neuroprotective when applied under clinical conditions $(12,13)$. For this reason, we investigated the effect of rEPO in a rabbit model of Gram-negative bacterial meningitis with rEPO administered from the onset of antibiotic treatment.

\section{MATERIALS AND METHODS}

Pathogen. An Escherichia coli K1-strain isolated from a child with neonatal meningitis (gift of Dr. G. Zysk, Institute of Medical Microbiology, Düsseldorf, Germany) was cultured on blood agar plates at $37^{\circ} \mathrm{C}$, harvested with $0.9 \%$ saline, and preserved in aliquots containing $10^{10}$ colony-forming units per milliliter $(\mathrm{CFUs} / \mathrm{mL})$ at $-70^{\circ} \mathrm{C}$. Minimal inhibitory concentration and minimal bactericidal concentration for ceftriaxone determined by broth microdilution were 0.0625 and $0.125 \mathrm{mg} / \mathrm{L}$, respectively.

Animal model. Animal experiments were performed in accordance with national animal experimentation guidelines and were approved by the District Government of Braunschweig, Lower Saxony. Anesthetized rabbits were infected by intracisternal injection of $300 \mu \mathrm{L}$ containing $\log _{10} 4.0$ CFUs (median, $\log _{10}$; range, 3.9-5.3 CFUs) E. coli. Cisternal cerebrospinal fluid (CSF) and arterial blood were drawn at $0,12,14,18$, and $24 \mathrm{~h}$. At each time point, CSF leukocytes were counted and bacterial titers were determined. The remaining CSF as well as the arterial blood anticoagulated with ethylenediamine tetraacetic acid were immediately centrifuged at $3000 \times g$ for $10 \mathrm{~min}$, and the supernatants were frozen in liquid nitrogen and stored at $-70^{\circ} \mathrm{C}$. At $23.5 \mathrm{~h}$ post-infection, an arterial blood gas analysis was performed (Radiom-

Abbreviations: CFU, colony-forming unit; CSF, cerebrospinal fluid; EPO, erythropoietin; rEPO, recombinant human erythropoietin; HE, hematoxylineosin (staining); NSE, neuron-specific enolase 
eter ABL 605, Diamond Diagnostics) to determine arterial oxygen $\left(\mathrm{PO}_{2}\right)$ and carbon dioxide tension $\left(\mathrm{PCO}_{2}\right)$ and $\mathrm{pH}$. The animals were killed at $24 \mathrm{~h}$ post-infection (14).

Treatment. Before infection, animals were randomized for treatment with rEPO or an equal volume of saline. At $12 \mathrm{~h}$ post-infection, all animals $(n=$ 26) received intravenous antibiotic treatment (ceftriaxone, $125 \mathrm{mg} / \mathrm{kg}$ body weight, Hoffmann LaRoche, Grenzach-Wyhlen, Germany). In 10 animals, rEPO (a kind gift of Janssen-Cilag, Neuss, Germany) was administered as bolus injection intravenously (1000 IU/kg body weight) at the time point of onset of antibiotic treatment and $6 \mathrm{~h}$ later. This dose was chosen in accordance with a study describing a neuroprotective effect in a rabbit model of subarachnoid hemorrhage (6)

Analysis of CSF. Protein content was quantified using the BCA Protein Assay (Pierce, Rockford, IL) with bovine serum albumin as standard according to the manufacturer's instructions. CSF lactate was determined enzymatically (Rolf Greiner Biochemica, Flacht, Germany). Plasma and CSF concentrations of EPO were quantified using a commercially available enzyme immunoassay (R\&D Systems, Minneapolis, MN). The global neuronal destruction marker neuron-specific enolase (NSE) was quantified in CSF by enzyme immunoassay with mouse anti-human monoclonal antibody against the $\gamma$-subunit of NSE (DRG Instruments, Marburg, Germany). CSF bacterial titers were quantified by plating serial 10 -fold dilutions on blood agar plates, which were subsequently incubated for $20 \mathrm{~h}$ at $37^{\circ} \mathrm{C}(15)$.

Histopathology. Immediately after sacrifice, the animals' brains were removed and fixed in $4 \%$ paraformaldehyde and embedded in paraffin. In situ tailing and hematoxylin-eosin (HE) staining were performed as described previously (16).

Quantification of apoptotic neurons. Four coronal sections from each rabbit's left hemisphere were histologically analyzed using the Analysis Software Imaging System (BX51; Olympus, Hamburg, Germany; software AnalySIS 3.2; Soft Imaging System GmbH, Münster, Germany). In coded sections, a blinded observer counted dentate granule cells labeled by in situ tailing. Adjacent sections stained by hematoxylin-eosin (staining) (HE) showed morphologic features of apoptosis in the same neurons. The density of apoptotic neurons was expressed as the number of marked cells per square millimeter of the granule cell layer of the hippocampal dentate gyrus, measured in the adjacent HE-stained section.

Statistical analysis. In the presence of normal gaussian distribution, parameters of two groups were compared using an unpaired $t$ test. In the absence of normal distribution, statistical analysis was performed using the two-tailed Mann-Whitney $U$ test; $p<0.05$ was considered significant.

\section{RESULTS}

Course of disease. In this rabbit model of E. coli meningitis, one of 10 in the rEPO-treated group and three of 16 rabbits in the control group died before the end of the experiment. Only animals surviving at least $23 \mathrm{~h}$ after infection were included into the analysis to avoid effects of survival time on the rate of neuronal apoptosis (10 animals in the rEPO-treated group and 14 in the control group). Animals dying prematurely did not differ substantially from the surviving animals with respect to CSF pleocytosis and bacterial load. Twelve hours after intracisternal inoculation of $E$. coli, rabbits developed full clinical presentation of meningitis [bacterial CSF load $\log _{10} 7.93 \mathrm{CFUs} / \mathrm{mL}(5.30-9.00)$ (median, min, max)] and septicemia [bacteriemia of $\log _{10} 3.00 \mathrm{CFUs} / \mathrm{mL}\left(\log _{10}\right.$ 1.48, 5.48 (median, min, max)] without relevant differences between the two treatment groups. Systemic inflammation parameters in E. coli meningitis including arterial $\mathrm{PO}_{2}, \mathrm{PCO}_{2}$, and $\mathrm{pH}$ at $23.5 \mathrm{~h}$ post-infection did not differ significantly between the rEPO-treated and the control group (Table 1).

CSF analysis. At $12 \mathrm{~h}$ after intracisternal inoculation of E. coli, all rabbits developed meningeal inflammation (pleocytosis, elevated CSF levels of protein and lactate, high bacterial CSF load; Table 1). There was no significant difference between the rEPO group and the controls before treatment. CSF leukocytes, protein, and lactate further increased between $12 \mathrm{~h}$ and $24 \mathrm{~h}$. The 24-h CSF protein and lactate concentrations did not differ between the rEPOtreated and the respective control animals. rEPO did not affect bacterial elimination (Table 1). Plasma and CSF levels of EPO were determined $18 \mathrm{~h}$ after infection. EPO levels of meningitic control animals $(n=3)$ treated with ceftriaxone only were below the detection limit of the assay at $6 \mathrm{mIU} / \mathrm{mL}$ in CSF and plasma. In the animals treated with intravenous $\mathrm{rEPO}$ at a dose of $1000 \mathrm{IE} / \mathrm{kg}$ body weight, EPO concentrations in CSF were $1291 \pm 568$ $\mathrm{mIU} / \mathrm{mL}(n=10)$ and $6993 \pm 1406 \mathrm{mIU} / \mathrm{mL}$ in plasma (mean $\pm \mathrm{SD}, n=9$ ) $6 \mathrm{~h}$ after injection. The CSF-to-plasma ratio of rEPO was $0.18 \pm 0.07$ in the rEPO-treated animals.

Global neuronal damage. NSE in CSF as an indicator of neuronal destruction was quantified at $12 \mathrm{~h}$ and $24 \mathrm{~h}$ after infection (Table 1) (14). The NSE concentration at $12 \mathrm{~h}$ and $24 \mathrm{~h}$ post-infection did not differ significantly between rEPOtreated and control animals (Table $1, p=0.60$ and 0.72 , respectively, both $U$ test).

Neuronal apoptosis in the hippocampal dentate gyrus. Neuronal apoptosis was regularly induced by E. coli menin-

Table 1. Parameters of CSF inflammation, global neuronal damage, and blood gas analysis in rEPO-treated rabbits and controls

\begin{tabular}{|c|c|c|c|}
\hline & Time after infection (h) & \multicolumn{2}{|c|}{ E. coli meningitis } \\
\hline Bacterial load (log CFUs/mL)* & 12 & $7.65(5.30-9.00)$ & $8.00(7.00-9.00)$ \\
\hline \multirow[t]{2}{*}{ Leukocytes $($ cells $/ \mu \mathrm{L}) *$} & 12 & $581.5(120-2261)$ & $1424(99-3783)$ \\
\hline & 24 & $9483(1578-26450)$ & $4992(501-61870)$ \\
\hline Protein $(\mathrm{mg} / \mathrm{mL})^{*}$ & 12 & $2750(1500-5600)$ & $2550(1100-4200)$ \\
\hline Lactate $(\mathrm{mmol} / \mathrm{L}) \dagger$ & 24 & $15.18( \pm 4.60)$ & $15.71( \pm 2.93)$ \\
\hline \multirow[t]{2}{*}{ NSE* } & 12 & $26.0(11.0-219.0)$ & $26.0(11.0-296.0)$ \\
\hline & 24 & $303.0(32.0-702.0)$ & $143.0(26.0-1060)$ \\
\hline Arterial $\mathrm{pH} \dagger$ & 23.5 & $7.57( \pm 0.11)$ & $7.53( \pm 0.06)$ \\
\hline Arterial $\mathrm{Po}_{2} \dagger$ & 23.5 & $105.8( \pm 12.7)$ & $121.6( \pm 27.7)$ \\
\hline Arterial $\mathrm{PcO}_{2} \dagger$ & 23.5 & $17.96( \pm 8.9)$ & $15.8( \pm 7.1)$ \\
\hline
\end{tabular}

* Median (minimum; maximum), no significant difference between rEPO-treated and respective control group, $p>0.05$ ( $U$ test).

$\dagger$ Mean $( \pm \mathrm{SD})$, no significant difference between rEPO-treated and respective control group, $p>0.05$ ( $t$ test). 


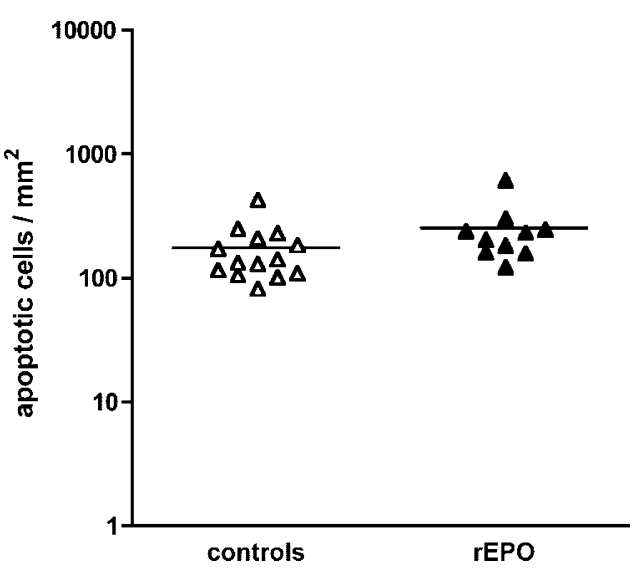

Figure 1. Rate of hippocampal apoptotic granule cells per area of the granule cell layer of the dentate gyrus in rabbit bacterial meningitis with or without adjunctive rEPO treatment. No significant difference was found in the rate of apoptotic neurons between rEPO-treated animals and respective controls. Symbols represent measurements of each individual animal (rEPO-treated $E$. coli meningitis: $\mathbf{\Delta}$, controls: $\Delta$ ), and horizontal bars represent means.

gitis in the granule cell layer of the dentate gyrus at $24 \mathrm{~h}$ after infection. Apoptotic cells were identified by in situ labeling of DNA double-strand breaks and confirmed by morphologic criteria (14). The rate of apoptotic neurons ranged from 85 to $633 / \mathrm{mm}^{2}$ (area of the granule cell layer, $208 \pm 120$, mean \pm $\mathrm{SD})$. Adjunctive rEPO treatment administered from the time point of antibiotic treatment did not reduce the rate of neuronal apoptosis in the hippocampal dentate gyrus in comparison to control animals ( $p=0.12$, unpaired $t$ test, Fig. 1).

\section{DISCUSSION}

Bacterial meningitis is a life-threatening infection of children and adults with high rates of neurologic sequelae including epileptic seizures, mental retardation, and learning disorders. Despite effective antibiotic treatment, stimulation of the host's systemic and local immune defense and direct toxicity of bacterial products result in impairment of the CNS. Neuropsychological deficits and cognitive sequelae in bacterial meningitis are at least in part caused by neuronal cell death detected in the hippocampal formation of patients (17). Bilateral hippocampal atrophy is seen by magnetic resonance imaging in patients who survived meningitis (18). In search of neuroprotective treatment strategies, we used a rabbit model of bacterial meningitis, which regularly exhibits neuronal apoptotic cell death in the granular cell layer of the hippocampal formation. In the absence of meningitis, the density of apoptotic neuronal cells $/ \mathrm{mm}^{2}$ in the hippocampal dentate gyrus was found to be approximately 10 apoptotic neurons $/ \mathrm{mm}^{2}$ in previously healthy rabbits killed immediately without longterm anesthesia (19). In uninfected control rabbits subjected to $24 \mathrm{~h}$ of urethane long-term anesthesia, antibiotic treatment, and repeated suboccipital punctures the density of apoptotic neuronal cells was approximately 40 apoptotic neurons $/ \mathrm{mm}^{2}$ of the dentate gyrus (14). The greatly increased rate of apoptotic neurons in the hippocampal dentate gyrus of rabbits suffering from meningitis (in the present study: $208 \pm 120$ apoptotic neurons $/ \mathrm{mm}^{2}$, mean $\pm \mathrm{SD}$ ) can be attributed to the infection and serves as a parameter for the evaluation of putatively neuroprotective therapeutic agents.

In search of neuroprotective treatment strategies, we evaluated the hematopoetic cytokine EPO. The recombinant human cytokine rEPO is currently one of the most promising neuroprotective agents: it has been shown to be beneficial in various models of neurologic diseases. Neuroprotective effects have been found in models of acute neuronal damage such as ischemic stroke (20), neonatal hypoxia-ischemia (21), traumatic brain injury (22), subarachnoid hemorrhage (6), and pilocarpine-induced status epilepticus (23). Likewise rEPO was neuroprotective in chronic models of neuroinflammation such as experimental autoimmune encephalomyelitis (10) or in a model of Parkinson's disease (24). In animal models, recombinant human EPO exhibited its neuroprotective properties in different mammalian species including rabbits (6). Hippocampal neuronal cells, which are especially vulnerable during bacterial meningitis, were also protected from neuronal death in animal models (23). This prompted us to investigate the neuroprotective effect of $\mathrm{rEPO}$ in experimental bacterial meningitis.

Many studies revealed neuroprotective effects of a variety of substances when applied in a narrow time window before or shortly after the induction of neuronal impairment but failed to be beneficial under clinical treatment conditions $(12,13)$. In clinical practice, the earliest time point of adjunctive treatment in bacterial meningitis is the time of a patient's admission to a medical center. Hence, in the present study, a design was chosen in which rEPO was administered from the time of antibiotic treatment, a treatment that should be administered as quickly as possible after diagnosing bacterial meningitis. To date, no data have been published on the pharmacokinetics of rEPO in bacterial meningitis. Only very few data are available about the penetration of EPO in the CSF in patients with noninflamed meninges (25) and in the brain tissue of rats after cerebral hypoxia (26). In our rabbit model of $E$. coli meningitis, we investigated the pharmacokinetics of rEPO and could demonstrate a high penetration rate with a CSF-to-plasma ratio of $0.18 \pm 0.07$ (mean $\pm \mathrm{SD}$ ) $6 \mathrm{~h}$ after injection.

Additional rEPO treatment did not affect the course of the disease in our model of E. coli meningitis in a statistically significant manner. One of 10 rabbits in the rEPO-treated group versus three of 16 rabbits in the control group died before the end of the experiment, i.e. mortality was not significantly different ( $p=1.00$, Fisher exact test). Even though we cannot fully exclude a type II error, we assume that no true difference in mortality was present. Accordingly, no significant differences could be found for parameters of inflammation in CSF or systemic circulation and for bacterial elimination (Table 1).

Despite high rEPO concentrations reached in the CSF in bacterial meningitis, no neuroprotective effect of rEPO was found under clinical treatment conditions, neither with respect to the rate of apoptotic neuronal cells in the hippocampal dentate gyrus nor to the global neuronal injury marker NSE in CSF.

In conclusion, no neuroprotective effect of rEPO could be found in this rabbit model of $E$. coli meningitis and therefore 
rEPO is not recommended as an adjunct to antibacterial therapy in human bacterial meningitis.

Acknowledgments. The authors thank Stefanie Bunkowski for excellent technical support.

\section{REFERENCES}

1. Nau R, Brück W 2002 Neuronal injury in bacterial meningitis: mechanisms and implications for therapy. Trends Neurosci 25:38-45

2. Morishita E, Masuda S, Nagao M, Yasuda Y, Sasaki R 1997 Erythropoietin receptor is expressed in rat hippocampal and cerebral cortical neurons, and erythropoietin prevents in vitro glutamate-induced neuronal death. Neuroscience 76:105-116

3. Siren AL, Fratelli M, Brines M, Goemans C, Casagrande S, Lewczuk P, Keenan S, Gleiter C, Pasquali C, Capobianco A, Mennini T, Heumann R, Cerami A, Ehrenreich H, Ghezzi P 2001 Erythropoietin prevents neuronal apoptosis after cerebral ischemia and metabolic stress. Proc Natl Acad Sci U S A 98:4044-4049

4. Digicaylioglu M, Lipton SA 2001 Erythropoietin-mediated neuroprotection involves cross-talk between Jak2 and NF-kappaB signalling cascades. Nature 412:641-647

5. Sättler MB, Merkler D, Maier K, Stadelmann C, Ehrenreich H, Bahr M, Diem R 2004 Neuroprotective effects and intracellular signaling pathways of erythropoietin in a rat model of multiple sclerosis. Cell Death Differ 11:S181-S192

6. Grasso G, Buemi M, Alafaci C, Sfacteria A, Passalacqua M, Sturiale A, Calapai G, De Vico G, Piedimonte G, Salpietro FM, Tomasello F 2002 Beneficial effects of systemic administration of recombinant human erythropoietin in rabbits subjected to subarachnoid hemorrhage. Proc Natl Acad Sci U S A 99:5627-5631

7. Genc S, Akhisaroglu M, Kuralay F, Genc K 2002 Erythropoietin restores glutathione peroxidase activity in 1-methyl-4-phenyl-1,2,5,6-tetrahydropyridine-induced neurotoxicity in $\mathrm{C} 57 \mathrm{BL}$ mice and stimulates murine astroglial glutathione peroxidase production in vitro. Neurosci Lett 321:73-76

8. Chattopadhyay A, Choudhury TD, Bandyopadhyay D, Datta AG 2000 Protective effect of erythropoietin on the oxidative damage of erythrocyte membrane by hydroxyl radical. Biochem Pharmacol 59:419-425

9. Villa P, Bigini P, Mennini T, Agnello D, Laragione T, Cagnotto A, Viviani B, Marinovich M, Cerami A, Coleman TR, Brines M, Ghezzi P 2003 Erythropoietin selectively attenuates cytokine production and inflammation in cerebral ischemia by targeting neuronal apoptosis. J Exp Med 198:971-975

10. Agnello D, Bigini P, Villa P, Mennini T, Cerami A, Brines ML, Ghezzi P 2002 Erythropoietin exerts an anti-inflammatory effect on the CNS in a model of experimental autoimmune encephalomyelitis. Brain Res 952:128-134

11. Kawakami M, Sekiguchi M, Sato K, Kozaki S, Takahashi M 2001 Erythropoietin receptor-mediated inhibition of exocytotic glutamate release confers neuroprotection during chemical ischemia. J Biol Chem 276:39469-39475
12. Grotta J 2002 Neuroprotection is unlikely to be effective in humans using current trial designs. Stroke 33:306-307

13. Muir KW, Teal PA 2005 Why have neuro-protectants failed? Lessons learned from stroke trials. J Neurol 252:1011-1020

14. Spreer A, Gerber J, Hanssen M, Schindler S, Hermann C, Lange P, Eiffert H, Nau R 2006 Dexamethasone increases hippocampal neuronal apoptosis in a rabbit model of Escherichia coli meningitis. Pediatr Res 60:210-215

15. Nau R, Zysk G, Schmidt H, Fischer FR, Stringaris AK, Stuertz K, Brück W 1997 Trovafloxacin delays the antibiotic-induced inflammatory response in experimenta pneumococcal meningitis. J Antimicrob Chemother 39:781-788

16. Böttcher T, Ren H, Goiny M, Gerber J, Lykkesfeldt J, Kuhnt U, Lotz M, Bunkowski S, Werner C, Schau I, Spreer A, Christen S, Nau R 2004 Clindamycin is neuroprotective in experimental Streptococcus pneumoniae meningitis compared with ceftriaxone. J Neurochem 91:1450-1460

17. Nau R, Soto A, Brück W 1999 Apoptosis of neurons in the dentate gyrus in humans suffering from bacterial meningitis. J Neuropathol Exp Neurol 58:265-274

18. Free SL, Li LM, Fish DR, Shorvon SD, Stevens JM 1996 Bilateral hippocampal volume loss in patients with a history of encephalitis or meningitis. Epilepsia 37:400-405

19. Zysk G, Brück W, Gerber J, Brück Y, Prange HW, Nau R 1996 Anti-inflammatory treatment influences neuronal apoptotic cell death in the dentate gyrus in experimental pneumococcal meningitis. J Neuropathol Exp Neurol 55:722-728

20. Bernaudin M, Marti HH, Roussel S, Divoux D, Nouvelot A, MacKenzie ET, Petit E 1999 A potential role for erythropoietin in focal permanent cerebral ischemia in mice. J Cereb Blood Flow Metab 19:643-651

21. Demers EJ, McPherson RJ, Juul SE 2005 Erythropoietin protects dopaminergic neurons and improves neurobehavioral outcomes in juvenile rats after neonata hypoxia-ischemia. Pediatr Res 58:297-301

22. Cherian L, Goodman JC, Robertson C 2007 Neuroprotection with erythropoietin administration following controlled cortical impact injury in rats. J Pharmacol Exp Ther 322:789-794

23. Nadam J, Navarro F, Sanchez P, Moulin C, Georges B, Laglaine A, Pequignot JM, Morales A, Ryvlin P, Bezin L 2007 Neuroprotective effects of erythropoietin in the rat hippocampus after pilocarpine-induced status epilepticus. Neurobiol Dis 25:412426

24. Kanaan NM, Collier TJ, Marchionini DM, McGuire SO, Fleming MF, Sortwell CE 2006 Exogenous erythropoietin provides neuroprotection of grafted dopamine neurons in a rodent model of Parkinson's disease. Brain Res 1068:221-229

25. Xenocostas A, Cheung WK, Farrell F, Zakszewski C, Kelley M, Lutynski A, Crump M, Lipton JH, Kiss TL, Lau CY, Messner HA 2005 The pharmacokinetics of erythropoietin in the cerebrospinal fluid after intravenous administration of recombinant human erythropoietin. Eur J Clin Pharmacol 61:189-195

26. Statler PA, McPherson RJ, Bauer LA, Kellert BA, Juul SE 2007 Pharmacokinetic of high-dose recombinant erythropoietin in plasma and brain of neonatal rats. Pediatr Res [Epub ahead of print] 